\title{
Caracterización ecológica y social de humedales altoandinos del Parque Nacional Huascarán
}

Ecological and social characterization of andean wetlands Huascaran National Park

\author{
Beatriz Fuentealba ${ }^{1}$ y Mayra MejíA ${ }^{1}$
}

\section{RESUMEN}

Este trabajo busca caracterizar cuatro humedales altoandinos ubicados en las quebradas Pucavado y Ulta, del Parque Nacional Huascarán. Para ello, hemos medido las características hídricas, edáficas y de la vegetación de cada humedal, e identificado los usuarios y usos determinados en cada quebrada. Nuestros resultados muestran tres tipos de humedales de acuerdo al régimen hídrico anual, la profundidad de la turba y pH. Además, se muestra la relación que existe entre el nivel de la napa freática y la abundancia de vegetación almohadillada, lo que a su vez influye en la densidad aparente y al contenido de materia orgánica en el suelo y la abundancia de especies vegetales que usamos como indicadoras de alteraciones. Estas variables parecen ser sensibles a cambios recientes, ya que puntos medidos en un mismo humedal muestran diferencias en estas características. En cuanto a los usuarios, encontramos que, en general, los principales servicios ecosistémicos percibidos son la provisión de agua y de forraje para el ganado, que son los principales usos que se les ha dado a estos humedales. Consideramos que la información generada es importante para definir las estrategias de recuperación de estos humedales, involucrando a la población local.

Palabras clave: bofedal; profundidad de turba; impactos de ganadería.

\begin{abstract}
In this research, we want to characterize four Andean Wetlands located in two areas (Pucavado and Ulta) of Huascaran National Park. Therefore, we took measures of plants, soil and water conditions, in several points in each wetland. Additionally, we identified the stakeholders and uses during last years, of these wetlands. Our results show three types of wetlands, based on water level annual variation, peat depth, and
\end{abstract}

1 Instituto de Montaña. Huaraz, Perú 
$\mathrm{pH}$. We also found that water level affect the abundance of cushion vegetation, and this relationship influence the bulk density and organic matter in the soil, and the abundance of plant species used as disturbance indicators. These variables apparently are sensitive indicators of current conditions, because their found differences between points in the same wetland. About stakeholders and uses, we found that local people perceive water and fodder for cattle provision as the most important ecosystem services. These are harvest in different ways in each area. We think that all this information will be important to design restoration actions, involving local people.

Keywords: andean peatland; cushion vegetation; cattle impacts.

\section{ICHIKLLACHAW}

Kay musyapakuyqa chusku uquykuna antikunachaw kanqantam ashin, kaykunaqa Pukawaduwan Ulta raqrankunachaw Parki Nasiyunal Waskaran nishqanchawmi karaayan. Tsaypaq yaku kanqanta, allpa kanqanta, wayllakuna uquchaw kayanqantam tupukashqa, hinaman llapan runakunapa raqrakunachaw kayanqantam riqikashqa. Kay musyapakuychaw riqitsikamun imanawmi kima laaya uquykuna huk watachaw kanqantam, hinamanpis rurinchawwan $\mathrm{pH}$ nishqan yarqamushqantam rikaatsikamun. Hinamanpis imanawmi napa freatrika nishqanwan atska quchuqashqa mallkikuna tinkuynin kanqantam rikaatsikamun. Hinamanpis llapan patsachaw kanqantawan atska tukuy laaya mallkikuna runakuna iñishiyanqan ichik tikraynintam. Kay llapan nishqantsik kanan qipa patsamanqa ichik tikrakaakuykanmi, kikin uquyllachaw tupushqa chikukuna huklaayashqanam yarqamun. Llapan wanaq runakunachaw yarqamun imanawmi purwakashqa llapan kawaqkuna rikakamun, imanawmi yaku quchakanqantawan uushakunapaq qiwankuna kanqantam riqitsimantsik. Kay uquykunallatam imaypis allaapa wanakashqa. Tsayraykurmi kay musyapakuy willakuyqa allaapa alli, tsaynawpa yapay uqukuna unay kanqanmannaw churanapaq, tsayman llapan markachaw taakuq runakunata yaykutsishpa.

Pushaq shimikuna: uqu; turbapa rurin; hatun waatakuy.

\section{INTRODUCCIÓN}

Los humedales se definen como áreas saturadas de agua dulce o salobre, bajo un régimen hídrico natural o artificial, permanente o temporal, y que albergan comunidades biológicas características (MINAM, 2015). Los bofedales, llamados también «oconales», son un tipo de humedal propio de las regiones altoandinas, ubicados por encima de los 3300 m.s.n.m. (Ibíd). Estos ecosistemas generalmente están dominados por plantas que forman almohadillados y acumulan turba (material vegetal en descomposición, también llamado suelo orgánico) (Cooper et al., 2010; Maldonado-Fonkén et al., 2015). En el Perú, el área que ocupan los bofedales se estima en 544562 ha (0.42 $\%$ del total nacional), (Loyola, 2015). 
Los humedales, en general, cumplen un rol ecológico muy importante. Por ejemplo, los humedales con escaso drenaje y acumulación de turba almacenan grandes cantidades de agua y de carbono (Maldonado y Maldonado, 2010). Se estima que las áreas con turba representan el 3\% de la superficie de la Tierra, pero almacenan el 19\% del carbono presente en todos los suelos (Batjes, 1996; Dise, 2009). Otras funciones importantes son la regulación y depuración hídrica, muy importante en las zonas altoandinas (Mostacero-León et al., 2008). Además, controla la erosión del suelo, regula el microclima alrededor del humedal y mantiene diversidad de fauna asociada (Oyague-Passuni y Maldonado-Fonkén, 2015; Florez, Tácuna y Calvo, 2014).

En el área altoandina, muchos humedales son reconocidos como importantes fuentes de agua para la población actual. Pero también lo eran para las poblaciones precolombinas, ya que se han encontrado construcciones hechas para regar y mantener las áreas con humedales (Lane, 2009). Asimismo, los bofedales son una fuente permanente de forraje para el ganado, incluso durante la época seca, y se puede extraer la turba para secar y usar como combustible, y/o para vender como abono para viveros (Cooper et al., 2010, Maldonado-Fonkén, 2014). Sin embargo, estos mismos usos, si no son bien manejados (i.e. sobrepastoreo o extracción excesiva de turba), se convierten en importantes amenazas para estas áreas. Estos se consideran ecosistemas frágiles por su lenta capacidad de respuesta, y cualquier alteración en su funcionamiento, especialmente en su hidrología, genera fuertes impactos y degradación (Benavides, 2014; Salvador et al., 2014).

En los humedales, en general, podemos diferenciar tres componentes principales: agua, suelo y vegetación. Las condiciones hidrológicas probablemente sean las más importantes, por ello, entender las variaciones que existen naturalmente en el nivel del agua o napa freática en un humedal es fundamental para comprender su funcionamiento. Se ha encontrado, por ejemplo, que el régimen hídrico puede cambiar drásticamente de acuerdo a la estación y a la variación anual de la precipitación (Carafa, 2009); mientras en otros casos se mantiene constante en el año.

En cuanto al suelo, se ha encontrado que muchos humedales altoandinos tienen uno o varios horizontes de turba (o suelo orgánico), que pueden llegar a medir en total desde centímetros hasta varios metros, y esto se relaciona con la antigüedad del humedal (Hribljan et al., 2015). Esta turba está compuesta por raíces vivas, muertas y abundante materia orgánica en descomposición que se va acumulando debido a las condiciones climáticas y a la baja presencia de oxígeno en el suelo (Cooper et al., 2010). Los suelos orgánicos, en comparación con los inorgánicos, tienen valores bajos de compactación, alta estabilidad y su pH tiende a ser más ácido (Salvador et al., 2014; Cooper et al., 2015).

La vegetación que crece en los humedales altoandinos necesita estar adaptada a las duras condiciones climáticas que ahí dominan (Maldonado y Maldonado, 2010). En el Perú, las especies que caracterizan la mayoría de bofedales son Distichia muscoides, Oxychloe andina (ambas de la familia Juncaceae), Plantago rigida y Plantago tubulosa (de la 
familia Plantaginaceae). Todas estas forman almohadillados y turba (Salvador et al., 2014). Sin embargo, se ha identificado otras comunidades vegetales también como bofedales. Por ejemplo, humedales altoandinos con alta presencia de especies de la Familia Poaceae (Festuca y Calamagrostis), y Cyperaceae (Carex, Eleocharis, Pbylloscirpus), a los que llama «prados turbosos». Y ambientes en que dominan los musgos del género Sphagnum y arbustos pequeños como Loricaria ferruginea y Vaccinium floribundum (Maldonado-Fonkén, 2014). Esto nos muestra que hay una gran heterogeneidad en la composición y estructura vegetal de los bofedales y humedales altoandinos en general.

Los humedales altoandinos son ecosistemas únicos, frágiles y que brindan importantes servicios ecosistémicos para la población local. Sin embargo, en el Perú, es muy poco lo que conocemos sobre su funcionamiento y estructura. Sólo entendiendo mejor la relación que existe entre los diferentes componentes de estos ecosistemas, y los impactos que generan los diferentes usos a los que son sujetos, podremos plantear alternativas de manejo, conservación y/o restauración. En este contexto, los objetivos de este trabajo fueron: a) Caracterizar la condición ecológica de cuatro humedales altoandinos ubicados dentro del Parque Nacional Huascarán e, b) Identificar los principales usuarios de esos humedales, y los principales usos que se les ha dado.

\section{MATERIALES Y MÉTODOS}

Esta es una investigación descriptiva, que busca contribuir al conocimiento de las características y funciones ecológicas en los humedales altoandinos, así como entender mejor a los usuarios y la forma de manejo de estas zonas. Estos resultados son parte del proyecto «Construyendo las bases científicas y sociales para la restauración de humedales altoandinos», desarrollado por el Instituto de Montaña desde octubre del 2014.

Área de estudio

El Parque Nacional Huascarán (PNH), creado en 1975, se ubica en el Departamento de Ancash (Perú), y abarca aproximadamente 340 mil hectáreas, entre los 2400 y 6768 msnm. (SERNANP, 2011). Dentro del PNH, el sobrepastoreo y las alteraciones hidrológicas (debido a la construcción de carreteras, de canales de drenaje y al retroceso glaciar) se ha identificado como las principales amenazas para los humedales altoandinos (O’Donnell, 2015). Sabiendo esto, y con apoyo del personal del PNH, se seleccionaron dos quebradas representativas de estas condiciones. Las quebradas elegidas fueron Pucavado, en el Distrito Chavín de Huántar, Provincia de Huari (Coordenadas UTM: 18L 254420 8928130) y Ulta, en el Distrito de Shilla, Provincia de Carhuaz (Coordenadas UTM: 18L 221893 8992483). 
En la quebrada de Pucavado, elegimos dos humedales (tabla 1): P1, en la parte alta, bastante homogéneo y aparentemente con poca alteración, y P2, muy heterogéneo, con alteraciones en su hidrología debido a la construcción de la carretera y a la presencia de varios canales de drenaje. En la quebrada Ulta, la principal alteración está dada por la alta densidad de ganado vacuno que se encuentra pastoreando libremente en la quebrada (observación personal). En esta quebrada también se eligieron dos humedales (Tabla 1): U1, ubicado en la parte alta, con vegetación almohadillada dominante, señales de sobrepastoreo y heterogeneidad en la humedad del suelo, y U2, ubicado en la parte baja, con menos humedad, dominancia de gramíneas y condiciones más homogéneas.

En cada humedal se establecieron puntos de muestreo. La cantidad de estos varió de acuerdo al componente evaluado (agua, suelo y vegetación, Tabla 1). En Pucavado, se diferenciaron 3 sitios conservados (incluyendo un sitio en P1), 3 sitios degradados (cercanos a un canal de drenaje), y 2 sitios muy degradados (con presencia de turba pero sin vegetación almohadillada). En U1 se buscó representar la heterogeneidad de humedad (2 sitios más secos, y 2 más húmedos), y en U2, el más homogéneo, se establecieron 4 puntos.

Para el componente hídrico, se colocaron tubos piezométricos (tubos de PVC de $5 \mathrm{~cm}$ de diámetro y al menos $100 \mathrm{~cm}$ de profundidad, con canales laterales que permitan el ingreso de agua) en cada punto de monitoreo. Y cada mes o mes y medio se midió el nivel de la napa freática, usando una cinta métrica acoplada a un voltímetro. En la mayoría de tubos piezométricos se tomaron medidas de $\mathrm{pH}$ y conductividad eléctrica usando un equipo multiparámetro (Hanna HI 9828).

En cuanto a la vegetación, se hizo una caracterización general usando dos o tres transectos de 50m en cada humedal, cada uno con 100 puntos de evaluación. También se colectaron las especies que no cayeron en los transectos para tener un listado completo de especies presentes en cada humedal. Además, en cada punto de monitoreo, se evaluó la vegetación usando cuadrantes de $1 \mathrm{~m}^{2}$ (100 puntos de evaluación), usando el método de intercepción puntual.

Para el análisis del suelo, se hicieron calicatas de 30x30 cm y de profundidad variable (según la profundidad de la turba presente) en áreas cercanas a los tubos piezométricos. Se describió el perfil del suelo, identificando horizontes y la profundidad de cada uno. Luego, con un cilindro metálico $(6 \times 6 \mathrm{~cm})$, se sacaron muestras del horizonte de turba más superficial (entre 0 y $15 \mathrm{~cm}$ ). Estas muestras fueron llevadas al Laboratorio de Agua y Suelos de la Facultad de Ciencias Agrarias - UNASAM, para calcular la densidad aparente y contenido de humedad. Además, se tiene los datos del contenido de materia orgánica, en el mismo horizonte superficial de los lugares donde se establecieron los tubos piezométricos. Estos datos fueron calculados y compartidos por Ana María Planas, estudiante de la Universidad Tecnológica de Michigan, Estados Unidos. 


\section{Análisis de datos}

Para la caracterización hidrológica, se utilizaron los datos de $\mathrm{pH}$, la profundidad promedio de la napa freática, a lo largo del año, y el tipo de régimen hídrico en cada tubo piezométrico evaluado. Para la categorización del régimen hídrico se revisaron los datos de un año completo y se consideró: a) Permanente, cuando el suelo estuvo saturado de agua todo el año, es decir, el nivel de la napa freática permaneció entre 0 y $20 \mathrm{~cm}$ de profundidad; b) Estacional, si es que la saturación del suelo se dio sólo durante los meses de lluvia; y c) Ocasional, si es que la saturación del suelo se dio uno o dos meses al año. Para la caracterización del suelo, se estimó la profundidad de la turba y se usó el porcentaje de materia orgánica y la densidad aparente del horizonte superficial.

En el caso de la vegetación, se usaron los datos de los transectos para estimar la riqueza de especies y la diversidad de cada humedal. Mientras que, la información de los cuadrantes se usó para estimar el porcentaje de cobertura vegetal, y el porcentaje que representan las diferentes formas de crecimiento, en las especies vegetales. Las formas de crecimiento consideradas fueron: almohadillados y musgo, pastos o gramíneas y hierbas (propuesta modificada de Naoki et al., 2014). Por último, se diferenciaron algunas especies indicadoras de disturbio, de acuerdo a la literatura y al conocimiento experto (Dámaso, Ramírez), y se calculó el porcentaje que representan. Las especies elegidas fueron: Werneria nubigena, Geranium sessiliflorum, Agrostis breviculmis, Mublenbergia ligularis, Poa annua, y Aciachne acicularis, ya que no son típicas de humedales, y/o que aumentan su abundancia cuando se alteran las condiciones.

Con esta información se buscó agrupar los puntos de muestreo, según las condiciones medidas. Para ello, se probó el nivel de correlación entre todas las variables medidas, y se eligieron las variables que más información daban de cada componente: nivel promedio de la napa freática (hidrológico), el porcentaje de materia orgánica (suelo), y el porcentaje de cobertura con almohadillados (vegetación). Con estas variables, normalizadas y estandarizadas, se hizo un análisis de conglomerados o Cluster, utilizando el método de Ward y el programa Past. Utilizando todas las variables medidas, previamente transformadas, se hicieron pruebas de regresión lineal, buscando relaciones significativas entre componentes.

\section{Identificación de usuarios y formas de uso}

Con apoyo del personal del PNH, se identificó a los usuarios de los humedales en cada quebrada. En noviembre del 2015 se realizaron dos talleres, uno con los usuarios de cada quebrada. En ambos talleres se reflexionó sobre la condición de sus ecosistemas antes (hace unos 20 años), ahora, y cómo querían verlos en el futuro. Con esto se reconocieron los usos pasados de los humedales caracterizados, y las formas de uso actual. Además, como parte de otro trabajo, se realizaron encuestas a parte de los usuarios de la quebrada Pucavado, y se incluyeron preguntas sobre la percepción del cambio, 
y la importancia de los humedales cercanos al caserío. Toda la información obtenida fue transcrita y organizada por quebrada, y se presentan los principales resultados de manera descriptiva.

\section{RESULTADOS}

A continuación se muestra los principales resultados de la caracterización ecológica de los cuatro humedales evaluados, incluyendo las similitudes y diferencias entre sitios y las relaciones encontradas entre las características del suelo, del agua y la vegetación. También se resume la información obtenida sobre los usuarios y usos de estos cuatro humedales.

\section{Caracterización ecológica}

Encontramos claras diferencias en las condiciones ecológicas de los humedales evaluados en la quebrada Pucavado y los de Ulta (tabla 1). Los primeros son humedales con turba profunda, $\mathrm{pH}$ más ácidos, y un menor número de especies vegetales; además son humedales alimentados por agua durante todo el año. En P1 el agua está al nivel del suelo todo el año (régimen hídrico permanente); en P2 hay sectores donde el agua está al nivel del suelo sólo en la época de lluvias (régimen hídrico estacional), y sectores con régimen permanente. Mientras que, en los humedales de Ulta la turba es más superficial, el pH es casi neutro y se registró un mayor número de especies vegetales. En U1, la mayoría de sitios tiene un régimen hídrico estacional, con un punto saturado permanentemente, y presenta turba más profunda que U2. En U2, el agua llega al nivel del suelo sólo algunos meses al año (régimen hídrico ocasional) y tiene la turba más superficial de todos los sitios.

Tabla 1. Características de los cuatro humedales altoandinos evaluados

\begin{tabular}{|c|c|c|c|c|c|c|c|c|c|}
\hline Sitio & $\begin{array}{l}\text { Área } \\
\text { (ha) }\end{array}$ & $\begin{array}{l}\text { Altitud } \\
\text { (msnm) }\end{array}$ & $\begin{array}{l}\text { Puntos } \\
\text { muestreo }\end{array}$ & $\begin{array}{l}\text { Profundi- } \\
\text { dad turba } \\
\quad(\mathrm{cm})\end{array}$ & $\begin{array}{c}\mathrm{pH} \\
\text { agua }\end{array}$ & $\begin{array}{l}\mathrm{N}^{\circ} \text { Es- } \\
\text { pecies }\end{array}$ & $\begin{array}{c}\% \text { spp } \\
\text { distur- } \\
\text { bio }\end{array}$ & $\begin{array}{l}\text { Diversidad } \\
\text { Shannon }\end{array}$ & $\begin{array}{l}\text { Régimen hí- } \\
\text { drico }\end{array}$ \\
\hline U1 & 8.0 & 4250 & $4 \mathrm{~A}, 8 \mathrm{~V}, 3 \mathrm{~S}$ & $20-30$ & 6.5 & 37 & 7.4 & 3.20 & Estacional \\
\hline $\mathrm{U} 2$ & $<1.0$ & 4030 & $4 \mathrm{~A}, 8 \mathrm{~V}, 2 \mathrm{~S}$ & $5-8$ & 6.5 & 36 & 40.7 & 3.26 & Ocasional \\
\hline P1 & 3.5 & 4420 & $2 \mathrm{~A}, 1 \mathrm{~V}, 1 \mathrm{~S}$ & $>100$ & 5.7 & 25 & 0 & 3.14 & Permanente \\
\hline P2 & 9.1 & 4200 & $10 \mathrm{~A}, 7 \mathrm{~V}, 5 \mathrm{~S}$ & $>100$ & 5.7 & 32 & 14.9 & 3.16 & $\begin{array}{l}\text { Estacional a } \\
\text { Permanente }\end{array}$ \\
\hline
\end{tabular}

P1 = Pucavado parte alta, P2= Pucavado parte media, U1= Ulta parte alta y U2= Ulta parte baja. Número de puntos de muestreo en que se evalúa $\mathrm{A}=$ agua, $\mathrm{V}=$ vegetación y $\mathrm{S}=$ suelo. $\%$ spp disturbio $=$ porcentaje promedio de frecuencia de especies indicadoras de disturbio 
En cuanto a la vegetación dominante, en P1 dominan especies almohadilladas (Distichia sp., Plantago tubulosa) y gramíneas (Festuca longivaginata, Calamagrostis tarmensis), y no hay presencia de especies indicadoras de disturbio. En P2 se encuentra una composición similar, pero $\sin$ P. tubulosa, se encuentra una mayor presencia de especies indicadoras de disturbio, y de Carex of ecuadorica y Oritrophium limnophilum. En U1 dominan las especies almohadilladas (Distichia muscoides, Oreobulus obtusangulus, Plantago tubulosa), y gramíneas (Calamagrostis rigescens), además de Werneria nubigena, hierba indicadora de disturbio. Mientras que en U2, encontramos principalmente gramíneas (Poa annua, Calamagrostis rigescens, Muhelenbergia ligularis) y Lachemilla pignata (hierba), y alta abundancia de especies indicadoras de disturbio. Los valores de diversidad calculados son similares entre sitios.

El análisis de conglomerados (figura 1) agrupa los puntos de muestreo con más similitudes, diferenciando tres grupos. En el grupo 1 están los puntos en que la napa freática es más superficial, y con mayor abundancia de vegetación almohadillada. Este grupo incluye los puntos «conservados» de la quebrada Pucavado (P1.1, P2.1 y P2.2), y todos los puntos de U1. El grupo 2, con valores intermedios de almohadillado y de la napa freática, encontramos los sitios degradados (P2.3, P2.5) y uno muy degradado (P2.9), de la quebrada Pucavado. Y en el grupo 3, están los puntos con poco almohadillado y la napa freática más profunda, que incluye los sitios de U2, y P2.10.

El sitio P2 muestra mayor heterogeneidad, ya que sus puntos de muestreo están distribuidos en los tres grupos formados. Y una revisión más detallada, nos muestra que U1.4 (círculo gris, figura 1) tiene una napa freática más superficial que U1.1 y U1.3, pero mayor proporción de suelo expuesto, y mayor densidad aparente. Mientras que P2.10 (círculo gris, figura 1), en comparación con los otros puntos de P2, tiene una mayor densidad aparente, mayor presencia de especies indicadoras de disturbio y una napa freática muchó más profunda.

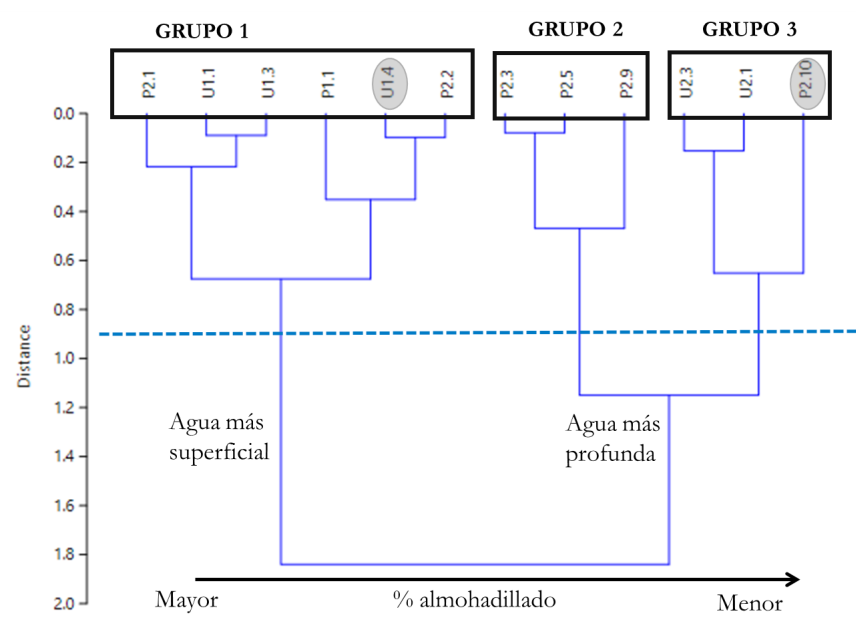

Figura 1. Grupos formados usando el análisis de conglomerados 
Por último, al buscar relaciones entre las variables, encontramos que cuando, en promedio, la napa freática es más superficial, la vegetación almohadillada es más abundante $\left(\mathrm{F}_{(1,11)}=8.5^{*}, \mathrm{p}<0.05, \mathrm{R}^{2}=0.36\right)$. Y cuando hay una mayor abundancia de vegetación almohadillada, la densidad aparente es menor $\left(\mathrm{F}_{(1,9)}=7.2, \mathrm{p}<0.05, \mathrm{R}^{2}=0.41\right)$, el porcentaje de materia orgánica, en la capa superior, aumenta $\left(\mathrm{F}_{(1,10)}=5.3, \mathrm{p}<0.05, \mathrm{R}^{2}=0.38\right)$, y la presencia de especies indicadoras de disturbio disminuye $\left(\mathrm{F}_{(1,11)}=8.1, \mathrm{p}<0.05, \mathrm{R}^{2}=0.37\right)$.

Usuarios y formas de uso

Dentro del Parque Nacional Huascarán (PNH) sólo tienen derecho de usufructo de los pastos y áreas de humedal, aquellas comunidades que existían previamente a la creación del Parque. Cada comunidad está representada por un Comité de Usuarios de Pastos Naturales (CUPN), que es la instancia que coordina acciones con el PNH. En la quebrada Ulta, el derecho de usufructo lo tiene el CUPN Huaypán, aunque la comunidad se encuentra a más de $8 \mathrm{Km}$ de Ulta. Esto es porque los comuneros han mantenido el derecho de usufructo de los terrenos que antes de la Reforma Agraria, eran parte de la hacienda ganadera de la actual comunidad de Huaypán.

De acuerdo a los miembros del CUPN Huaypán, la quebrada Ulta siempre ha sido usada para el pastoreo del ganado, principalmente vacuno, aprovechando las áreas con pastos naturales y los humedales. El hato ganadero total de la quebrada es difícil de determinar. Sin embargo, de acuerdo a los datos del CUPN, cuando se crea el PNH había 312 usuarios, con aproximadamente 5 cabezas de ganado por persona. En el 2015 se registraron 141 usuarios, con 3 o 4 cabezas de ganado por persona. Aunque con valores diferentes, el PNH a través de los rodeos anuales, también registra una disminución en el ganado desde su creación (datos sin publicar).

El ganado que usa los humedales estudiados en la quebrada Ulta ha recibido y recibe muy poco manejo, por lo que se ha vuelto «chúcaro» (salvaje). Antes, los usuarios aprovechaban el rodeo del PNH, para llevar a su ganado y venderlo; pero ahora no pueden sacar al ganado de la quebrada. Por ello, los miembros del CUPN desde este año han empezado a reemplazar el ganado «chúcaro» por un menor número de vacas de razas cruzadas, pero que sean mejor manejadas. Así esperan recuperar parte del beneficio económico que antes recibían. Sobre los humedales (u «oconales» que es como se los conoce en la zona), los miembros del CUPN consideran que se han ido reduciendo porque, por efecto del cambio climático, hay menor disponibilidad de agua y de precipitación, además las heladas ahora son más frecuentes. Asimismo, señalan que los humedales son importantes por ser áreas de pastoreo del ganado.

En cuanto a la quebrada de Pucavado, los usuarios son pequeños propietarios que habitan los caseríos de Shirapata y Tambillos. Ambos caseríos se fundaron mucho antes de la reforma agraria y ahora se encuentran dentro de los límites del PNH. Los humedales que estudiamos, son parte de las tierras de usufructo de Shirapata. 
Los pobladores de Shirapata señalan que P1 no se ha pastoreado nunca, solo en las orillas, porque tiene mucha agua y es peligroso para los animales. Mientras que P2, fue un área de pastoreo hasta hace unos 5 años en que se retiró todo el ganado, porque no hay quien lleve al ganado hasta la zona (P2 se encuentra a $4.4 \mathrm{Km}$ del caserío). La mayoría de gente que reside en Shirapata tiene 60 o más años. Sin embargo, los pobladores de Tambillos mantienen la actividad ganadera, y a veces llevan vacas y ovejas a pastorear a P2, lo que revive conflictos históricos que han tenido estos dos caseríos.

Los pobladores de Shirapata indican que antes los oconales eran más extensos, y la vegetación aún más exuberante, pero los cambios en el clima, la construcción de la carretera, entre otros, han afectado la cantidad y calidad del agua, así como de la abundancia de vegetación. Ellos, además, mencionan que los canales de drenaje que hay en P2 son naturales; y que el sobrepastoreo pudo afectar las áreas de pastos, pero no identifican impactos en los humedales.

En general, la población reconoce que los oconales son importantes reservas de agua, y fuente importante de forraje para los animales durante todo el año. Sin embargo a la mitad de los encuestados no le preocupaba el deterioro de estas áreas, porque ya no reciben un beneficio directo, ya que no tienen animales en la zona. La otra mitad si se mostró interesada en la conservación de los oconales como fuente de agua limpia, y una posible actividad turística.

\section{DISCUSIÓN}

Nuestros resultados muestran que, aún en condiciones naturales, hay una gran variabilidad en las características de los humedales altoandinos. En este caso, reconocer el régimen hídrico y la profundidad de la turba ha sido fundamental para diferenciar los tres tipos de humedal encontrados. Apoyando lo que otros autores han demostrado, que el nivel de la napa freática determina en gran medida el funcionamiento y composición del humedal (Hooijer et al., 2012 Benavides et al., 2014). Los sitios con turba más profunda, tienen una mayor saturación de agua, lo que genera un ambiente anaeróbico y favorece la lenta descomposición del mantillo. Por esta razón consideramos que, en ambientes con un régimen hídrico estacional, la turba encontrada sería producto de la acumulación durante la época de lluvias, y descomposición durante la época seca, cuando la napa freática es más profunda (Hribljan et al., 2015). Mientras que, un ambiente que se solo se satura de agua algunas semanas al año (régimen hídrico ocasional), podría funcionar como humedal y tal vez acumular turba sólo durante años atípicos y muy lluviosos. Y aunque son sólo dos sitios, nuestros resultados concuerdan con que los humedales con turba o suelo orgánico más profundo, presentan $\mathrm{pH}$ más ácidos (Salvador et al., 2014; Cooper et al., 2015). 
Encontramos también una gran variación en la composición y especies vegetales dominantes en los diferentes tipos de humedal. Sin embargo, las formas de vida dominantes no varían tanto. P1, P2 y U1 están dominadas por especies almohadilladas y gramíneas, y corresponderían a una turbera de Distichia, combinada con prados turbosos, de acuerdo a la clasificación propuesta por Maldonado-Fonkén (2014). Mientras que U2, no sería un bofedal, de acuerdo a las definiciones dadas (Maldonado-Fonkén, 2014; MINAM, 2015). Pero, además, encontramos que la presencia de vegetación almohadillada es un indicador del buen funcionamiento del humedal, inversamente relacionado con la abundancia de especies indicadoras de disturbio. Igual que Salvador et al. (2014), encontramos que los sitios con mayor abundancia de vegetación almohadillada, tienen una mayor concentración de materia orgánica y menor compactación. Y para mantener una buena abundancia de vegetación almohadillada, es necesaria una napa freática superficial.

Nuestros resultados, además, parecen demostrar que el $\mathrm{pH}$, profundidad de turba y régimen hídrico anual, son indicadores de procesos naturales más antiguos, ya que los valores varían más entre humedales que dentro de cada humedal. Mientras que, la abundancia de vegetación almohadillada, la materia orgánica y densidad aparente en la capa superficial, y el promedio del nivel de la napa freática parecen ser indicadores sensibles a los cambios recientes en el humedal. Para estas variables encontramos importantes diferencias dentro de un mismo humedal. En el sitio P2.10, se comprueba la relación entre napa freática y composición vegetal señalada por Benavides et al. (2014). Aquí, por alteraciones hidrológicas, la napa freática es muy profunda y actualmente no muestra vegetación almohadillada, y tiene menos materia orgánica y mayor compactación que otros sitios de P2. Mientras que U1.4, parece ser más afectada por el ganado que los otros sitios de U1, aunque no encontramos otros estudios que nos permitan comparar nuestros resultados. En U1.4 la napa freática es más superficial, con mayor cobertura de suelo expuesto, menos vegetación almohadillada y mayor compactación.

Finalmente, de acuerdo a la percepción de la población local, el cambio climático y la reducción de la precipitación son los principales factores que están reduciendo la extensión de los humedales. Además, los principales servicios que brindan los humedales son agua y forraje para el ganado. Esto debería ser tomado en cuenta para proponer estrategias de conservación y/o recuperación más efectivas. Tendríamos que entender mejor los impactos que genera el sobrepastoreo sobre los humedales, para concientizar a la población local. Y a su vez, deberíamos reconocer cómo los servicios hidrológicos y de forraje mejoran al recuperar un humedal altoandino. 


\section{CONCLUSIONES}

Los resultados demuestran que hay una alta heterogeneidad en las condiciones naturales de los humedales altoandinos, tanto en sus características hídricas y edáficas como en la composición vegetal. En este caso diferenciamos tres tipos de humedal, usando como indicadores principales el régimen hídrico anual y la profundidad de turba.

Se encontraron encontrado características que parecen ser más sensibles al disturbio reciente, como la abundancia de vegetación almohadillada (muy relacionada con el nivel actual de la napa freática), el contenido de materia orgánica en la capa superior de turba y la densidad aparente.

verifico que las especies elegidas como indicadoras de disturbio son adecuadas, ya que tienen una relación inversa con la abundancia de vegetación almohadillada. En cuanto a los aspectos sociales, encontramos que en el área de estudio, los principales beneficios percibidos por la población local son el agua y el forraje para el ganado. Finalmente, hemos encontrado algunos indicios de que el sobrepastoreo no afecta de igual forma todas las áreas del humedal.

\section{AGRADECIMIENTOS}

Este proyecto ha sido financiado por US Forest Service. Y queremos agradecer a Ana María Planas por compartir la información de contenido de materia orgánica, a Dámaso W. Ramírez por el apoyo en la identificación de la vegetación y a los voluntarios que nos acompañaron a campo durante todo el proceso, especialmente a Yanett Gonzáles y Edwin Giraldo. Un agradecimiento a Isabel Menéndez por sus comentarios y sugerencias para este artículo.

\section{REFERENCIAS BIBLIOGRÁFICAS}

Batjes, Niels. 1996. «Total carbon and nitrogen in the soils of the world ». European Journal of Soil Science, Vol 47, N². 151-163.

Benavides Juan. 2014. "The effect of drainage on organic matter accumulation and plant communities of high-altitude peatlands in the Colombian tropical Andes». Mires and Peat 15.1-15

Carafa, Tania. 2009. Evaluación ecológica de los bofedales de la cuenca circundante al nevado Illimani. La Paz: Proyecto Agua sustentable.

Cooper David, y otros. 2010. «Alpine peatlands of the Andes, Cajamarca, Peru». Arctic, Antarctic, and Alpine Research 42 N¹. 19-33. 
Cooper David, y otros. 2015. «Growth and organic carbon production in peatlands dominated by Distichia muscoides, Bolivia, South America». Arctic, Antarctic, and Alpine Research Vol. 47, N³ 505-510.

Dise, Nancy. 2009. «Peatland response to Global Change». Science Vol. 326. 810-811.

Flóres, Enrique, Rául Tácuna, y Vivian Calvo. 2014. Marco conceptual y metodológico para estimar el estado de salud de los Bofedales. Lima: Laboratorio de utilización de pastizales - Universidad Nacional Agraria la Molina.

Hooijer Aljosja y otros. 2012. «Subsidence and carbon loss in drained tropical peatlands». Biogeosciences, Vol. 9, N³ 1053-1071

Hribljan, John y otros. 2015. «Carbon storage and long-term rate of accumaltion in high-altitude Andean peatlands of Bolivia». Mires and Peat, Vol 15. 1-14.

Instituto de Montaña (IM). 2016. Resultados del diagnóstico en la comunidad de Shirapata. Reporte interno.

Lane Kevin. 2009. «Engineered highland: the social organization of water in the ancient north-central Andes (AD 1000-1480)» World Archaeology Vol. 41, N¹ 169190.

Loyola, Roger et al. 2015. Mapa Nacional de Cobertura Vegetal. Lima: Ministerio del Ambiente. Dirección General de Evaluación, Valoración y Financiamiento del Patrimonio Natural.

Maldonado-Fonkén, Mónica, y L. Daniel Maldonado. 2010. «Los bofedales: Un oasis en la puna». En Anuario Cultural. Huaraz: Antamina. 70-77.

Maldonado-Fonkén Mónica. 2014. «An introduction to the bofedales of the Peruvian High Andes». Mires and Peat Vol. 15. 1-13.

MINAM. 2015. Estrategia Nacional de Humedales. Documento para consulta pública, Lima: Ministerio del Ambiente Dirección General de Diversidad Biológica.

Mostacero-León, José; Ramírez, Rosa y Mejía Freddy. 2008. «Caracterización biológica, física y química de los humedales altoandinos de La Libertad. Perú». REBIOL, Vol. 28. 91-98.

Naoki, kazuya y otros. 2014. «El uso del método de puntos de intercepción para cuantificar los tipos de vegetación y hábitats abióticos en los bofedales altoandinos. Ecología en Bolivia». Ecología en Bolivia, Vol. 49, N³. 84-90.

O’Donnell, Collen. 2015. Taller de Humedales Alpinos Resumen y resultados. Huaraz: Instituto de Montaña. 
Oyague-Passuni Eduardo y Maldonado-Fonkén. 2015. «Relationships between aquatic invertebrades, water quality and vegetation in an Andean peatland system». Mires and Peat Vol. 15. 1-21.

Salvador Flor, Monerris, Jorge y Rochefort, Line. 2014. Peatlands of the Peruvian puna ecoregion: types, characteristics and disturbance. Mires and Peat Vol. 15. 1-17.

SERNANP. 2011. Parque Nacional Huascarán Plan Maestro 2010-2015. Lima: SERNAMP.

Fecha de recepción: 10 de junio de 2016

Fecha de aceptación: 22 de noviembre de 2016

\section{Correspondencia}

Dr. Beatriz Fuentealba

bfuentealba@mountain.org 